\title{
Ruptura diafragmática por trauma contuso en un hospital del noreste de México para referencia de pacientes politraumatizados: reporte de caso y revisión de la literatura
}

\author{
Traumatic diaphragmatic injury in a hospital at Northeastern Mexican for reference of \\ polytraumatized patients: case report and review of the literature
}

\author{
Vianey A. Hernández-Arteaga ${ }^{1 *}$, William's L. López-Vidal ${ }^{1}$ y Ricardo A. Garza-Treviño ${ }^{2}$ \\ ${ }^{1}$ Departamento de Emergencias Shock Trauma, Hospital Universitario Dr. José Eleuterio González; ${ }^{2}$ Facultad de Medicina. Universidad Autónoma \\ de Nuevo León, Monterrey, Nuevo León, México
}

\section{Introducción}

Cada año mueren aproximadamente 1.35 millones de personas por accidentes de tránsito y en promedio 3700 personas pierden la vida cada día por la misma razón en el mundo․ Se considera que los accidentes por vehículos con motor son la principal causa de muerte en adolescentes y adultos jóvenes globalmente ${ }^{2}$. La gravedad de los accidentes automovilísticos depende de una amplia variedad de factores, incluyendo la altura del paciente, la edad, la sobriedad, la posición del asiento ${ }^{3}$ y sobre todo el uso del cinturón de seguridad, el cual se ha relacionado con una disminución de las lesiones fatales de hasta 8 veces ${ }^{4}$.

El diafragma es un músculo en forma de domo que divide la región torácica de la abdominal y es el principal motor de la ventilación. Las rupturas diafragmáticas son más comunes en pacientes con heridas por debajo del tórax, presentándose en un 10-15\%; en cambio, la incidencia de las rupturas por trauma contuso es del $0.8-7 \% \%^{5}$. En la mayoría de los pacientes con ruptura diafragmática se encuentran lesiones asociadas, principalmente fractura de pelvis $y$ traumatismo craneoencefálico, que varían en frecuencia según el tipo de impacto; por lo tanto, la sospecha diagnóstica sobre estas lesiones no es la primera causa que descartar en los pacientes con este tipo de traumas ${ }^{6}$

Reportamos el caso de una paciente de 40 años que sufrió un accidente de tránsito y arriba al área de urgencias por un trauma contuso toracoabdominal.

\section{Presentación del caso}

Mujer de 40 años que arriba al servicio de urgencias de nuestro hospital mediante ambulancia tras participar en un accidente automovilístico de alto impacto. Los paramédicos refieren choque frontal contra un muro de contención, así como volcadura del vehículo con muerte de los acompañantes en el lugar. La paciente ocupaba el lugar del copiloto y no portaba cinturón de seguridad, por lo que salió proyectada 4 metros aproximadamente. Arriba al área de choque, con 6 puntos en escala de coma de Glasgow (M4 O1 V1). En la exploración física se observa contusión frontal derecha, pupilas $2 \mathrm{~mm}$ ojo derecho y $1 \mathrm{~mm}$ ojo izquierdo, sin reflejo corneal; no presenta heridas

\section{Correspondencia:}

*Vianey A. Hernández-Arteaga

E-mail: vale_arte8990@ hotmail.com
Fecha de recepción: 14-12-2021

Fecha de aceptación: 21-12-2021 DOI: 10.24875/REIE.21000314
Disponible en internet: 07-03-2022 Rev Educ Investig Emer. 2022;4(Supl 1):105-108 www.medicinadeemergencias.com bajo la licencia CC BY-NC-ND (http://creativecommons.org/licenses/by-nc-nd/4.0/). 


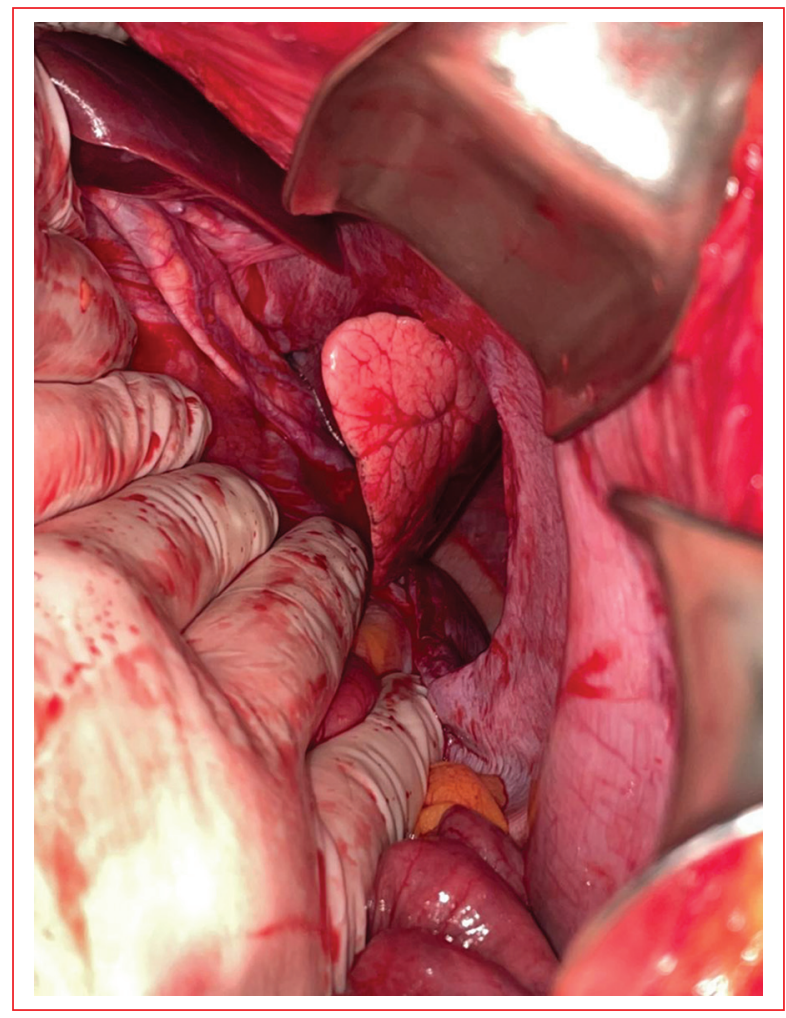

Figura 1. Lesión del hemidiafragma izquierdo de $12 \mathrm{~cm}$.

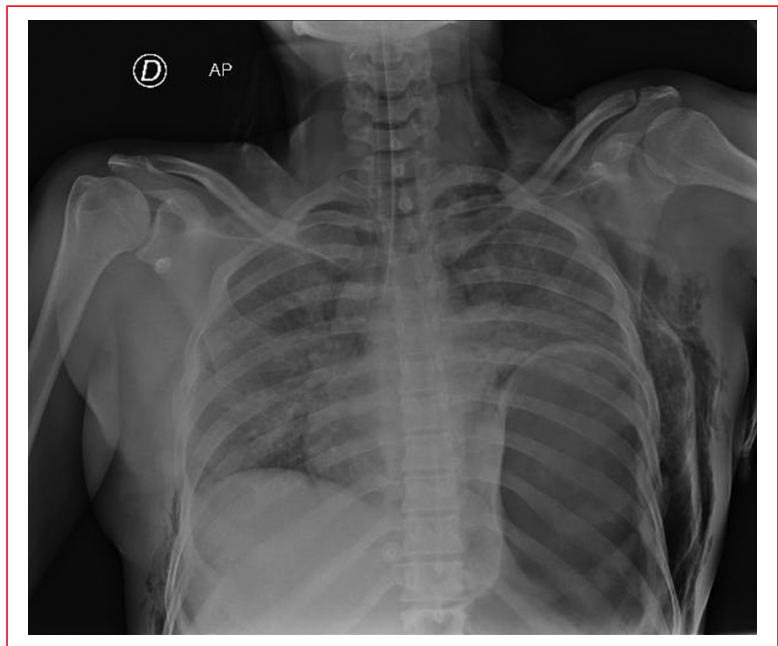

Figura 2. Radiografía de tórax: Se observa la burbuja gástrica en hemitórax izquierdo.

penetrantes, solo tatuaje de trauma contuso en el abdomen; a la palpación, enfisema subcutáneo en tórax bilateral, la expansibilidad torácica estaba disminuida en el hemitórax izquierdo, el murmullo vesicular se encontraba abolido en los dos tercios inferiores (donde

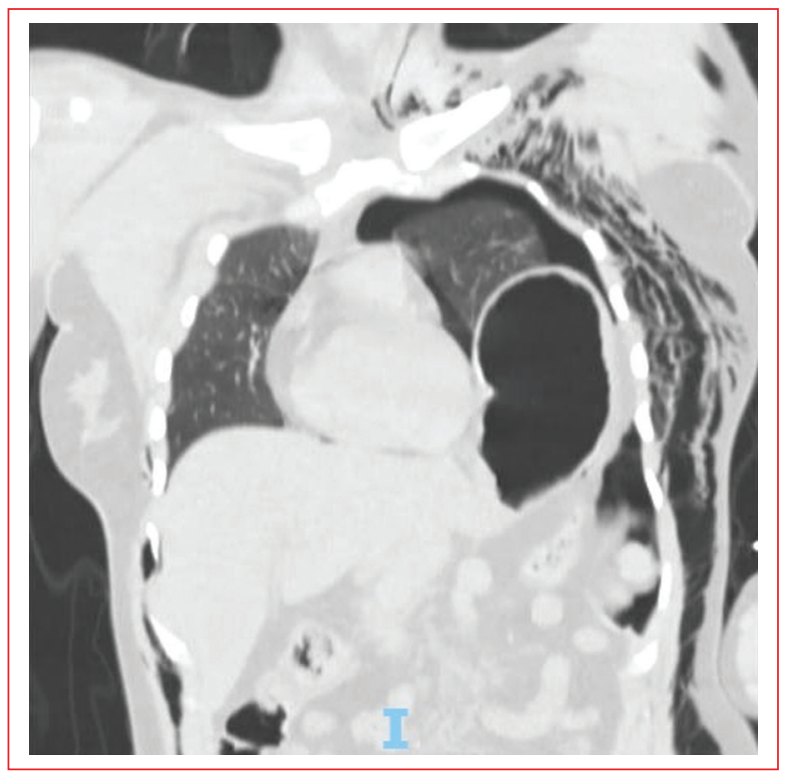

Figura 3. Tomografía de tórax: confirma el diagnostico al observarse lesión en hemidiafragma izquierdo.

se auscultaban ruidos hidroaéreos) y a la percusión había timpanismo en dicha región. Se decide manejo avanzado de la vía área, intervención que no presenta ninguna dificultad, y se colocan dos accesos vasculares de grueso calibre para la reanimación con líquidos.

\section{Abordaje}

Continuando con la evaluación de la paciente se ordena una serie radiográfica de trauma, en donde llama la atención en la imagen de burbuja gástrica en el hemitórax izquierdo, a nivel del quinto y el sexto espacios intercostales, además del signo de «alas de gaviota» con elevación de los hemidiafragmas (Fig. 2). Al momento se consideró que las imágenes observadas fueron debidas a una hiperinsuflación iatrogénica por la preoxigenación para la intubación. Se observan fractura isquiopúbica izquierda y múltiples fracturas costales, razón por la cual se deja en observación a la paciente en la sala de urgencias y se continúa con su estabilización. Se realiza tomografía computarizada en cortes axiales de la base a la convexidad y se observa una imagen hiperdensa menor de $1 \mathrm{~cm}$ a nivel de mesencéfalo izquierda, contusión mesencefálica.

Durante su abordaje en el área de choque se transfunden dos paquetes globulares de urgencias; se 
mantiene bajo sedación, con doble analgesia, y se inician vasopresores.

Una hora posterior al ingreso de la paciente, continúa con el deterioro de su estado hemodinámico, por lo que se decide nuevamente tomar una radiografía anteroposterior de tórax, donde continúa la presencia de la burbuja gástrica en el quinto espacio intercostal en el hemitórax izquierdo. Por ello, se ordena una tomografía computarizada simple de tórax, donde se hace evidente la herniación de vísceras y se decide realizar una laparotomía de urgencia (Fig. 3).

\section{Diagnósticos diferenciales}

Los estudios de imagen, los datos clínicos y la cinemática del trauma pueden hacer sospechar un neumotórax a tensión. Aunque el abordaje inicial debe ser el mismo en ambas situaciones, sin embargo, la gravedad y la resolución tienen que ser enfocadas de una manera más específica. Este caso en particular no cumple con los síntomas clásicos de un cuadro clínico de neumotórax a tensión, pero debe tomarse en cuenta como parte de los diagnósticos diferenciales.

\section{Tratamiento}

En la cirugía se observa una lesión de grado 5 del hemidiafragma izquierdo, de $12 \mathrm{~cm}$, con herniación del estómago, el bazo y asas intestinales, por lo que se realiza reparación quirúrgica y se coloca una sonda en el tórax (Fig. 1). Durante la cirugía se inicia protocolo de transfusión masiva con dos paquetes globulares, tres concentrados plaquetarios y dos plasmas frescos congelados. Al egreso de quirófano se mantiene hemodinámicamente inestable, continuando el abordaje en el área de cuidados intensivos.

\section{Desenlace y seguimiento}

Se traslada a la paciente a la unidad de cuidados intensivos debido a su estado crítico, donde se mantiene bajo ventilación mecánica invasiva, sedación y analgesia. En su décimo día de estancia intrahospitalaria se realizan traqueostomía y gastrostomía, y se inicia alimentación con suplemento. Con tendencia notoria a la mejoría, 3 días posterior a este procedimiento se logra el cese de la ventilación mecánica, es trasladada a piso, donde cumple días de vigilancia, y se egresa por mejoría clínica.

\section{Discusión}

En este caso nos enfocamos en el diagnóstico oportuno de una ruptura diafragmática por trauma. Esta lesión se presenta en menos del $0.5 \%$ de todos los casos de trauma, la mayoría de las veces causada por un mecanismo penetrante (67\%), seguido de lesiones contusas $(33 \%)^{7}$. La fisiopatología de este tipo de lesión no está bien entendida, pero la hipótesis más aceptada es un aumento de la presión intraabdominal siguiendo a un mecanismo contundente que crea un gradiente de presión suficientemente alto entre el tórax y el abdomen para causar la ruptura y la posterior hernia intratorácica visceral ${ }^{8}$. Los signos y síntomas más comunes son disnea, dolor torácico, distensión abdominal y pérdida de ruidos respiratorios sobre el hemitórax afectado ${ }^{9}$. En los accidentes de tránsito en vehículo con motor, una de las principales causas de ingreso a nuestro hospital, siempre debemos contemplar esta lesión como un diagnóstico diferencial. Hay que iniciar el abordaje con estudios de imagen; entre las de más fácil acceso está la radiografía de tórax, donde la imagen podría confundirse con datos de neumotórax a tensión, por lo que el interrogatorio y la cinemática del trauma son los que nos harán tener sospecha de este cuadro. En el área de urgencias nos enfrentamos ante pacientes que presentan alteración del estado de alerta, por lo que no será fácil realizar un interrogatorio dirigido. El diagnóstico tardío puede resultar en una morbilidad y mortalidad significativas ${ }^{10}$.

\section{Agradecimientos}

Agradecemos al personal del Departamento de Emergencias Shock Trauma por su colaboración durante la atención de esta paciente y su facilidad para llevar a cabo esta investigación.

\section{Financiamiento}

Los autores declaramos no haber recibido financiación para la realización de esta investigación.

\section{Conflicto de intereses}

Los autores declaramos no tener conflictos de intereses para la publicación y divulgación de esta investigación. 


\section{Responsabilidades éticas}

Protección de personas y animales. Los autores declaran que para esta investigación no se han realizado experimentos en seres humanos ni en animales.

Confidencialidad de los datos. Los autores declaran que han seguido los protocolos de su centro de trabajo sobre la publicación de datos de pacientes.

Derecho a la privacidad y consentimiento informado. Los autores han obtenido el consentimiento informado de los pacientes y/o sujetos referidos en el artículo. Este documento obra en poder del autor de correspondencia.

\section{Bibliografía}

1. Seid M, Azazh A, Enquselassie F, Yisma E. Injury characteristics and outcome of road traffic accident among victims at Adult Emergency Department of Tikur Anbessa specialized hospital, Addis Ababa, Ethiopia: a prospective hospital based study. BMC Emerg Med. 2015;15:10.
2. Fadl SA, Sandstrom CK. Pattern recognition: a mechanism-based approach to injury detection after motor vehicle collisions. Radiographics. 2019;39:857-76.

3. Febres JD, García-Herrero S, Herrera S, Gutiérrez JM, López-García JR Mariscal MA. Influence of seat-belt use on the severity of injury in traffic accidents. Eur Transp Res Rev. 2020;12:9.

4. Mihos P, Potaris K, Gakidis J, Paraskevopoulos J, Varvatsoulis P Gougoutas B, et al. Traumatic rupture of the diaphragm: experience with 65 patients. Injury. 2003;34:169-72.

5. Shaban Y, Elkbuli A, McKenney M, Boneva D. Traumatic diaphragmatic rupture with transthoracic organ herniation: a case report and review of literature. Am J Case Rep. 2020;21:e919442.

6. Brito G, Iraizoz AM. Catastrophic traumatic diaphragmatic hernia with subtotal section of the pylorus: case report. An Sist Sanit Navar. 2020;43:411-5.

7. Morgan BS, Watcyn-Jones T, Garner JP. Traumatic diaphragmatic injury. J R Army Med Corps. 2010;156:139-44.

8. Xiao J-C, Ma L-Y, Li B-L. Late traumatic diaphragmatic rupture complicated by haemothorax and strangulation of the stomach: a case report. J Int Med Res. 2020;48:300060520930864.

9. Allan Z, Peng C, Chandra R. Traumatic diaphragmatic rupture with underlying lung laceration and tension pneumoperitoneum. J Surg Case Rep. 2017;2017:rjx120.

10. Fair KA, Gordon NT, Barbosa RR, Rowell SE, Watters JM, Schreiber MA Traumatic diaphragmatic injury in the American College of Surgeons National Trauma Data Bank: a new examination of a rare diagnosis. Am J Surg. 2015;209:864-8; discussion 868-9. 